\title{
First principles model of internal transport barriers in negative central shear discharges
}

\author{
A.L. Rogister \\ Institut für Plasmaphysik, Forschungszentrum Jülich, Euratom Association, \\ Trilateral Euregio Cluster, Jülich, Germany
}

\begin{abstract}
At and around minima or maxima of the safety factor $q$, overlapping, and therefore toroidicity induced coupling of drift eigenmodes centred on neighbouring rational surfaces $r_{l, m}$ defined by $q\left(r_{l, m}\right)=m / l$ and $r_{l, m \pm 1}$ defined by $q\left(r_{l, m \pm 1}\right)=(m \pm 1) / l$, is generally negligible; $l$ and $m(m \pm 1)$ are the toroidal and (main) poloidal mode numbers, respectively. Under these conditions, a careful analysis shows that the growth rate of the ion temperature gradient (ITG) mode is proportional to the absolute value of the magnetic shear parameter; its radial width and frequency (in the $\boldsymbol{E} \times \boldsymbol{B}$ rotating frame) are larger in the axisymmetric torus than in the plasma slab or cylinder, especially at small values of $k_{\theta} a_{i}$ ( $k_{\theta}$ is the poloidal mode number and $a_{i}$ the characteristic ion Larmor radius). These results provide a straightforward theoretical interpretation of the origin of internal ion transport barriers (ITBs) in discharges with negative central shear if the ITG instability is indeed the main channel for ion energy transport. In agreement with recent experiments, the mechanism does not degrade for $T_{e} \sim T_{i}$, an important result for extrapolation to reactors. Once initiated, an ITB will locally increase the curvature of the ion temperature profile and, consequently, the gradients of the radial electric field and $\boldsymbol{E} \times \boldsymbol{B}$ rotation profiles (until neoclassical (or sub-neoclassical) transport comes into play). The damping associated with velocity shear yields an upper bound on the unstable mode number range, whereas Landau damping is expected to introduce a lower bound. In cases where toroidal effects are subdominant, the ratio of the damping rate associated with velocity shear to the growth rate associated with magnetic shear leads naturally to the Hamaguchi-Horton parameter (divided by the factor $\eta_{i}-2 / 3$ which characterizes the departure from the instability threshold $\left(\eta_{i}\right.$ is the ratio of the density and temperature length scales)) when introducing the most unfavourable mode number. Toroidal effects are always dominant for sufficiently weak magnetic shear or sufficiently large values of $\left|L_{N}\right| / R$ (where $L_{N}$ is the density gradient length), and therefore before steepening of the profiles has occurred near the zero magnetic shear layer; the counterpart of the Hamaguchi-Horton characteristic parameter appropriate to that case is also obtained, considering again the most unfavourable mode number.
\end{abstract}

\section{Introduction}

Analysis of negative central shear (NCS) tokamak discharges has shown that an ion internal transport barrier (ITB) may develop near the position where the safety factor $q$ reaches its minimum [1-6]. There is also evidence that, in addition to the NCS $q$ profile, $\boldsymbol{E} \times \boldsymbol{B}$ flow shear in the core plays an important role in stabilizing turbulence [7-9]. Our aim here is to demonstrate that these observations are easily understood in the framework of the theory of ion temperature gradient (ITG) instability - the latter being regarded as the main mechanism of ion energy losses - which is appropriate to the specific experimental conditions.

According to simple theoretical considerations, the behaviour of low frequency oscillations (including the ITG ones), or drift waves, in tokamaks is different according to whether the radial width of the 'elementary' eigenmodes is smaller or larger than the distance between the corresponding neighbouring rational surfaces. An elementary drift wave with toroidal and (main) poloidal mode numbers $l$ and $m$, respectively, is a radially bounded solution of a two dimensional eigenvalue equation which is localized in the vicinity of the rational surface defined by $q\left(r_{l, m}\right)=m / l$. Owing to the variation in the strength of the tokamak confining magnetic field with poloidal angle, the elementary modes centred on the neighbouring surfaces $r_{l, m}$ and $r_{l, m \pm 1}$ will interact and mutually modify their dynamics in the case of overlapping. The theory of ITG instability has been developed mainly with the help of the 'ballooning' formalism [10-12] under the hypothesis of large overlap. The poloidal position where ballooning occurs and the value of the growth rate depend on the choice of the phase relation between the elementary modes [13]; implicitly, this choice has generally been 
so as to maximize the instability growth rate. The distance between neighbouring rational surfaces is $\Delta_{l, m}=\left|r_{l, m \pm 1}-r_{l, m}\right|=|m(d \ln q / d r)|^{-1}=\left|1 / k_{\theta} \hat{s}\right|$, where $k_{\theta}=m / r$ is the poloidal mode number, $\hat{s}=r(d \ln q / d r)$ the magnetic shear parameter and, as the modes are strongly localized on the macroscopic scale, $q$ and $\hat{s}$ are evaluated at the radius $r=$ $r_{l, m}$. Since $\Delta_{l, m}$ becomes infinite (on the microscale under consideration) at positions where the $q$ profile reaches an extremum, overlapping and therefore interaction of 'neighbouring' elementary drift eigenmodes is impossible there.

\section{Initiation of ion ITBs in NCS discharges}

We have shown recently that, in the limit of negligible overlap, the eigenmode centred on the rational surface defined by $q\left(r_{l, m}\right)=m / l$ is necessarily of the form

$\Phi_{l}(x, \theta ; t ; r)=\hat{\Phi}_{l, m}(x, \theta) \exp [i(-m \theta+l \varphi-\omega t)]$

with $\partial \hat{\Phi}_{l, m}(x, \theta) / \partial \theta \neq 0[14] ; \varphi$ and $\theta$ are the toroidal and poloidal angles, respectively; $x=r-r_{l, m}$ is the distance from the rational surface. The $\theta$ dependence of the amplitude $\hat{\Phi}_{l, m}(x, \theta)$ can be described by a primary oscillation $(p=0)$ and a series of sidebands $(p= \pm 1, \ldots)$; thus

$\hat{\Phi}_{l, m}(x, \theta)=\sum_{p}\left[\hat{\Phi}_{l, m}(x)\right]_{p} \exp (i p \theta)$.

In the limit presently under consideration, the parallel mode number of the sidebands $p= \pm 1$ is larger than that of the primary component $p=0$ by the ratio $\Delta_{l, m} / w_{l, m} \gg 1$, where $w_{l, m}$ (given explicitly later) is the radial scale of the oscillation: the roles of the parallel ion dynamics and of waveparticle resonant interactions are therefore increased in the toroidal plasma geometry compared with the cylindrical plasma geometry. It can be shown that only the sidebands $p= \pm 1$ play an important role when the density length scale $L_{N}=(d \ln N / d r)^{-1}$ is smaller than the magnetic shear length $L_{s}=q R / \hat{s}$ (as is usually the case). The appropriate two dimensional linear fluid equations (these describe the fluctuations of density, parallel flow velocity and temperature; the limitations of the model are discussed in Section 4) can then be transformed into three times as many one dimensional equations. These can finally be reduced, after elimination of two of the field functions, into a single radial eigenvalue equation. The latter is, considering specifically the ion drift branch [14],

$\left[D \Delta_{\perp}-\left(\eta_{i}-2 / 3\right)^{-1} \omega^{\prime}-\lambda^{2}\left(\omega^{\prime}\right)^{-1}\left(x^{\prime}\right)^{2}\right]\left(\hat{t}_{i}\right)_{0}=0$

where $x^{\prime}=x / a_{i}, \omega^{\prime}=\left(\omega-\omega_{E \times B}\right) / \omega_{i}^{*}, \eta_{i}=$ $d_{r} \ln T_{i} / d_{r} \ln N_{i}, \lambda=L_{N} / L_{s},\left(\hat{t}_{i}\right)_{0}$ is the amplitude of the primary $(p=0)$ component of the normalized ion temperature oscillation,

$D=1+12.5\left(1+\tau_{e}^{-1}\right)\left[\varepsilon_{N} /\left(1.5 \eta_{i}-1\right) k_{\theta}^{\prime}\right]^{2}$

$k_{\theta}^{\prime}=k_{\theta} a_{i}, \varepsilon_{N}=L_{N} / R$ and $\tau_{e}=T_{e} / T_{i}$; the characteristic ion Larmor radius is defined as $a_{i}=c_{i} / \Omega_{i}$, where $c_{i}=\left(T_{i} / m_{i}\right)^{1 / 2}$ and $\Omega_{i}=e_{i} B / m_{i} ; \omega_{\boldsymbol{E} \times \boldsymbol{B}}$ is the $\boldsymbol{E} \times \boldsymbol{B}$ Doppler frequency shift and $\omega_{i}^{*}$ is the ion diamagnetic frequency. The operator $\Delta_{\perp} \equiv$ $\partial_{x^{\prime}}^{2}-k_{\theta}^{\prime 2}$ arises from finite ion Larmor radius (FLR) effects, whereas $(D-1) \Delta_{\perp}$ is a consequence of the $\cos \theta$ and $\sin \theta$ dependences of the $\nabla \boldsymbol{B}$ and curvature drifts. The eigenfunctions of Eq. (2) are readily obtained in the general case. It can be shown that there is at most one bounded, but marginally stable, branch if $\eta_{i}<2 / 3$; if $\eta_{i}>2 / 3$, there is, on the contrary, a pair of spatially bounded branches, one decaying, the other growing in time. Analysis of the latter solutions reveals that the two fluid model is inadequate if $w_{l, m}<k_{\theta}^{-1}$ owing to important wave-particle resonant interactions (those arise when $\left(\omega-\omega_{\boldsymbol{E} \times \boldsymbol{B}}\right) / k_{\|} c_{i} \leq 1$ and damp the modes; $k_{\|}=\boldsymbol{k} \cdot \boldsymbol{B} / B$ is the parallel mode number). Assuming therefore that $w_{l, m}>k_{\theta}^{-1}$ and $|\hat{s}|$ is sufficiently small that the inequality $w_{l, m} / \Delta_{l, m}=\hat{s} k_{\theta} w_{l, m}<1$ remains valid, the pair of bounded ground states obtained for $\eta_{i}>2 / 3$ is given by

$$
\begin{gathered}
\left(\hat{t}_{i}\right)_{0} \propto \exp \left[\mp i\left(\eta_{i}-2 / 3\right)^{-1 / 2}\left|\lambda / k_{\theta}^{\prime} D\right|\left(x^{\prime 2} / 2\right)\right. \\
\left.-\left(\eta_{i}-2 / 3\right)^{-1}\left(\lambda / k_{\theta}^{\prime} D\right)^{2}\left(x^{\prime} / 2 k_{\theta}^{\prime}\right)^{2}\right]
\end{gathered}
$$

(the whole series of orthogonal eigenmodes involves Hermite polynomials). Equation (4) describes a radial oscillation with weak spatial decay; the oscillation scale is

$w_{l, m}=\left(\eta_{i}-2 / 3\right)^{1 / 4}\left|D k_{\theta}^{\prime} / \lambda\right|^{1 / 2} a_{i}$

as the assumption $w_{l, m}>k_{\theta}^{-1}$ requires the imaginary part of the argument in the exponential (4) to be larger than the real part. The imaginary parts of the two complex conjugate eigenvalues $\omega^{\prime}$ yield the growth $\left(\gamma_{m}>0\right)$ and damping $\left(\gamma_{m}<0\right)$ rates,

$\gamma_{m}=\left(\mp \operatorname{sign} \omega_{i}^{*}\right)\left(\eta_{i}-2 / 3\right)^{1 / 2}|\hat{s}| c_{i} / q R$ 
whereas their real part yields the (common) real frequency

$\operatorname{Re}\left(\omega-\omega_{\boldsymbol{E} \times \boldsymbol{B}}\right)=-\left(\eta_{i}-2 / 3\right) D k_{\theta}^{2} a_{i}^{2} \omega_{i}^{*}$.

The parallel mode numbers of the primary oscillation and of the sidebands are $\left(k_{\|}\right)_{0} \cong k_{\theta} w_{l, m} / L_{s}$ and $\left(k_{\|}\right)_{p= \pm 1} \cong 1 / q R$, respectively. The condition of negligible wave-particle resonant interaction with either the former or the latter ( $\Longleftrightarrow$ in the inequality below), on the one hand, and the condition $\hat{s} k_{\theta} w_{l, m}<1$ of negligible overlap, on the other hand, impose the following lower and upper bounds on the fluctuation spectrum covered by the analysis:

$$
\begin{gathered}
\left(\eta_{i}-1 / 2\right)^{-1 / 2}\left|\varepsilon_{N} \hat{s} / q\right| \Longleftrightarrow\left|\varepsilon_{N} / q\right| \leq\left(\eta_{i}-2 / 3\right) D\left|k_{\theta}^{\prime}\right|^{3} \\
\quad \leq\left(\eta_{i}-2 / 3\right)^{1 / 2}\left|\varepsilon_{N} / q \hat{s}\right| .
\end{gathered}
$$

The following observations can be made at this stage:

(a) The requirement of negligible overlap (given by the right hand side inequality in (7)) is de facto fulfilled if $|\hat{s}| \rightarrow 0$.

(b) The growth rate vanishes if $|\hat{s}| \rightarrow 0$. It follows that an ITB will be initiated near the position of minimum $q$ if the ITG mode is the main channel of ion energy transport.

(c) The growth rate is independent of the temperature ratio.

(d) The constraint (7) depends on $\tau_{e}=T_{e} / T_{i}$ via the coefficient $D$ in such a manner that the range of validity of the assumption $w_{l, m}<$ $\Delta_{l, m}$, and therefore of the expression (6a) of the growth rate, extends to higher poloidal mode numbers if $T_{e} / T_{i}$ increases. This favourable trend (we note that there is a rapid increase of the growth rate when the condition $w_{l, m}<\Delta_{l, m}$ is no longer fulfilled) explains why transport barriers, first observed in plasmas with predominantly ion heating and characterized by $\tau_{e}<1$, are maintained when $\tau_{e} \sim 1$ [6]. This result is important for reactors with predominant heating by thermonuclear $\alpha$ particles.

\section{Creation of strong $E \times B$ rotation shear and synergy}

Our derivation of the ITG eigenvalue equation is 'local' in that it does not take the variations of the radial electric field and of the temperature and density gradients into account (these are not independent, as will be seen later). It is usually accepted that an ITG eigenmode will be torn apart and disintegrate before it can grow to a deleterious level if the variation, across its characteristic radial scale, of the Doppler frequency associated with the $\boldsymbol{E} \times \boldsymbol{B}$ rotation is comparable to, or larger than, the growth rate predicted by local theory, i.e. if [15]

$\left|w_{m} d \omega_{\boldsymbol{E} \times \boldsymbol{B}} / d r\right| \geq \gamma_{m}$.

This criterion reads here

$\left|E_{r}^{\prime} / B\right| \geq\left(\eta_{i}-2 / 3\right)^{1 / 4}\left|L_{N} /\left(D k_{\theta}^{\prime 3} L_{s}^{3}\right)\right|^{1 / 2} c_{i}$.

The right hand side of the above inequality decreases monotonically as $k_{\theta}^{\prime}$ increases, indicating that modes with higher poloidal mode numbers are stabilized first by the $\boldsymbol{E} \times \boldsymbol{B}$ rotation shear. At low mode numbers, the unstable spectrum is expected to disappear once wave-particle resonant interactions come into play. We shall assume wave-particle resonant interaction with the sidebands to be subdominant when the toroidal contribution to $D \Delta_{\perp}$ is also negligible, and vice versa. Below, we consider successively the two limiting cases.

\subsection{Toroidal effects can be neglected}

Comparison of inequality (8) and the reverse of the first left hand inequality (7) shows that the entire ITG spectrum is damped if

$\left|E_{r}^{\prime} / B\right| \geq\left(\eta_{i}-2 / 3\right) c_{i} /\left|L_{s}\right|$

(here we let $D \rightarrow 1$, in line with the above assumption). Moreover, the 'last marginally unstable' mode has the following poloidal mode number:

$\left|k_{\theta}^{\prime}\right|_{m . u .}=\left(\eta_{i}-2 / 3\right)^{-1 / 2}\left|L_{N} / L_{s}\right|^{1 / 3}$.

Inserting the latter into the requirement $D \rightarrow 1$, namely

$12.5\left(1+\tau_{e}^{-1}\right)\left[\varepsilon_{N} /\left(1.5 \eta_{i}-1\right) k_{\theta}^{\prime}\right]^{2} \ll 1$

further yields

$\left|\varepsilon_{N}\right| \ll 0.276\left(\eta_{i}-2 / 3\right)^{3 / 4}\left(1+\tau_{e}^{-1}\right)^{-3 / 4}|\hat{s}|^{1 / 2} q^{-1 / 2}$.

The ratio $\left|L_{s} E_{r}^{\prime}\right| / B c_{i}$, which is distinctive in criterion (9a), is the Hamaguchi-Horton dimensionless parameter. Inequality (11a) is an indication of the range of $\left|\varepsilon_{N}\right|$ for which the cylindrical model $(D \rightarrow 1)$ holds and for which the Hamaguchi-Horton parameter is relevant. This range vanishes when $|\hat{s}| \rightarrow 0$, decreases when $q$ increases and increases together with $\tau_{e}$. 


\subsection{Cylindrical effects can be neglected}

It follows from inequality (8) and the reverse of the second left hand inequality (7) that the entire ITG spectrum is now damped if

$\left|E_{r}^{\prime} / B\right| \geq\left(\eta_{i}-2 / 3\right)^{3 / 4}|\hat{s}|^{1 / 2} c_{i} /\left|L_{s}\right|$

(here we let $D \gg 1$ ); moreover, the 'last marginally unstable' mode has the poloidal mode number

$\left|k_{\theta}^{\prime}\right|_{m . u .}=0.18\left(\eta_{i}-2 / 3\right)\left(1+\tau_{e}^{-1}\right)^{-1} R / q\left|L_{N}\right|$.

(Note that the validity of the theory requires that $q R /\left|\hat{s} L_{N}\right| \gg 1 ; R /\left|L_{N}\right|$ may be finite.) Inserting (10b) into the condition $D \gg 1$, namely,

$12.5\left(1+\tau_{e}^{-1}\right)\left[\varepsilon_{N} /\left(1.5 \eta_{i}-1\right) k_{\theta}^{\prime}\right]^{2} \gg 1$

yields

$\left|\varepsilon_{N}\right| \gg 0.276\left(\eta_{i}-2 / 3\right)\left(1+\tau_{e}^{-1}\right)^{-3 / 4} q^{-1 / 2}$.

We note that:

(a) Criterion (9b) is more easily satisfied than criterion (9a), owing to the enhanced role of ion Landau damping on the sidebands and the larger radial scale of the modes if $D \gg 1$ (the wider toroidal modes are more fragile with respect to shearing). When toroidal effects dominate, the parameter $|\hat{s}|^{-1 / 2}\left|L_{s} E_{r}^{\prime} / B c_{i}\right|$ should be considered in lieu of the Hamaguchi-Horton parameter $\left|L_{s} E_{r}^{\prime} / B c_{i}\right|$. It is certainly enlightening to recall here that, in their original article, Hamaguchi and Horton solved numerically the linear two fluid eigenvalue equation of the plasma slab, in which the effects of both shear flow and Landau damping (the latter via a suitable model) were taken into account [16].

(b) Inequality (11b) yields the range of $\left|\varepsilon_{N}\right|$ for which toroidal effects play the leading role; this range, in contrast to that defined by (11a), is independent of $|\hat{s}|$ (thus it remains finite if $|\hat{s}| \rightarrow 0$ ), increases together with $q$ and decreases if $\tau_{e}$ increases.

(c) Finally, in the intermediate range of $\left|\varepsilon_{N}\right|$ defined by

$$
\begin{aligned}
|\hat{s}|^{1 / 2} & <3.62\left[\left(1+\tau_{e}^{-1}\right) /\left(\eta_{i}-2 / 3\right)\right]^{3 / 4} q^{1 / 2}\left|\varepsilon_{N}\right| \\
& <\left(\eta_{i}-2 / 3\right)^{1 / 4}
\end{aligned}
$$

the stability criterion should smoothly interpolate between (9a) and (9b).

\subsection{Synergetic effects}

In order to clarify the implications of criteria (9a) and $(9 \mathrm{~b})$, we introduce the radial momentum balance relation,

$E_{r}=B_{\theta} U_{\varphi, i}-B_{\varphi} U_{\theta, i}+\left(T_{i} / e_{i}\right) \partial_{r} \ln P_{i}$

where $U_{\theta, i}$ and $U_{\varphi, i}$ are the poloidal and toroidal flow velocities, respectively. A result of conventional neoclassical theory is that $U_{\theta, i}=\kappa\left(T_{i} / e_{i} B_{\varphi}\right) \partial_{r} \ln \mathrm{T}_{\mathrm{i}}$, where the coefficient $\kappa$ varies from 1.17 in the low collisionality (banana) regime to -1.83 in the high collisionality (Pfirsch-Schlüter) regime; thus

$E_{r}-B_{\theta} U_{\varphi}=\left(-\kappa+1+\eta_{i}^{-1}\right) \partial_{r} T_{i} / e_{i}$.

Neglecting the gradient of the toroidal velocity in order to simplify the argument, criteria (9a) and (9b) become

$$
\begin{gathered}
a_{i} q R T_{i}^{-1} \partial_{r}\left[\left(-\kappa+1+\eta_{i}^{-1}\right) \partial_{r} T_{i}\right] \\
\geq\left(\eta_{i}-2 / 3\right)|\hat{s}|
\end{gathered}
$$

and

$$
\begin{gathered}
a_{i} q R T_{i}^{-1} \partial_{r}\left[\left(-\kappa+1+\eta_{i}^{-1}\right) \partial_{r} T_{i}\right] \\
\geq\left(\eta_{i}-2 / 3\right)^{3 / 4}|\hat{s}|^{3 / 2}
\end{gathered}
$$

respectively.

An internal ITB will naturally modify the temperature profile locally, generating back to back negative and positive curvatures. (This suggests that the geometrical centre of the fully developed barrier could shift slightly from the position where $|\hat{s}|=0$ if the curvatures are different on both sides.) A most important consequence of the approximate criteria $\left(9 \mathrm{a}^{\prime}\right)$ and $\left(9 \mathrm{~b}^{\prime}\right)$ is therefore that a barrier, once initiated, is a self-reinforcing process. The increase of $\left|T_{i}^{\prime}\right|$ within the barrier will ultimately be limited by neoclassical $[17,18]$ or, if the gradients are particularly large, sub-neoclassical $[19,20]$ heat transport. The role of collisionality should be emphasized here: the factor $-\kappa+1+\eta_{i}^{-1}$ equals $-0.17+\eta_{i}^{-1}$ in the banana regime, whereas $-\kappa+1+\eta_{i}^{-1}$ equals $+2.83+\eta_{i}^{-1}$ in the Pfirsch-Schlüter regime! (The larger radial electric field and electric field gradient in the latter case may help to explain the improved confinement in high density radiative discharges (the so-called radiative improved confinement mode [21]); that problem will be discussed in another article, as the weak magnetic shear limit is not applicable). 


\subsection{Applicability of criteria (9a) and (9b)}

The parameter ranges covered by criteria (9a) and (9b) are specified in (11a) and (11b), respectively. To illustrate quantitatively these conditions and their relevance before steepening of the temperature profile (and of the density profile: we compare identical values of $\eta_{i}$ ) has occurred, let us assume that $T_{i}(r) \propto\left[1-(r / a)^{2}\right]^{2}, N_{i}(r) \propto\left[T_{i}(r)\right]^{1 / \eta_{i}}, B=2.5 \mathrm{~T}$, $T_{i}(r=0)=2 \mathrm{keV}, R=2 \mathrm{~m}, a=0.5 \mathrm{~m}$ and a deuterium plasma. At $r=a / 2\left(\right.$ where $L_{N}=0.75 \eta_{i} a$ ), inequalities (11a) and (11b) read (assuming $q=2$ and negligible variations of both $\kappa$ and $\eta_{i}$ )

$\left(\eta_{i}-2 / 3\right)^{3 / 4}|\hat{s}|^{1 / 2} \gg 0.96 \eta_{i}\left(1+\tau_{e}^{-1}\right)^{3 / 4}$

and

$\eta_{i}-2 / 3 \ll 0.96 \eta_{i}\left(1+\tau_{e}^{-1}\right)^{3 / 4}$

respectively. It is obvious from the above that criterion $(9 \mathrm{~b})$ is the relevant one in the early phase of development of ITBs in NCS discharges, at radii where $|\hat{s}| \cong 0$. In fully developed ITBs, i.e. after steepening has occurred, an interpolation formula between (9a) and (9b) needs in general to be used across the layer, in view of the $\left|\varepsilon_{N}\right|$ term appearing on the left hand sides of (11a) and (11b).

\section{Discussion}

Experimentally, electron energy transport often remains anomalous in NCS discharges. This is also in line with our earlier theoretical results [14] if electron drift waves are responsible for the electron heat flow anomaly; magnetic shear damping of the electron mode in toroidal plasmas indeed remains weak under conditions of negligible mode overlap if $|\hat{s}| \ll 1$ :

$\gamma_{m}=-\left(1+2 q^{2}\right)^{1 / 2}\left(1+\tau_{e}+\eta_{i}\right)\left|k_{\theta}^{\prime} \| \hat{s}\right| c_{i} / q R$

where the factor $\left(1+2 q^{2}\right)^{1 / 2}$ is a consequence of toroidicity $[14,22]$; we recall that instability is driven by trapped electrons, not included here. However, experimentally, the oscillation amplitude drops sharply over the entire drift wave range (including both the electron and ion branches) at the position of the ITB [23, 24], suggesting that anomalous electron heat transport is a consequence of either the electron temperature gradient (ETG) instability [25] or magnetic turbulence.

The conclusions of the present work are as follows:

(a) ITBs in NCS discharges can easily be explained on the basis of the theory of the ITG instability in the appropriate limit [14] (the distance between rational surfaces $\Delta_{l, m}$ is large in comparison with the radial extent of the modes $w_{l, m}$; we note, in this connection, that our analytical results agree qualitatively with numerical results obtained for weak magnetic shear [26] and small values of $k_{\theta} a_{i}$ [27].

(b) The cylindrical model is not relevant for explaining the early phase of ITB development (before steepening of the temperature and density profiles has occurred around the position where $\hat{s}=0$ ); moreover, an interpolation between the asymptotic criteria $(9 \mathrm{~b})$ and $(9 \mathrm{a})$ is generally required over the finite width of fully developed ITBs $((9 \mathrm{~b})$ and $(9 \mathrm{a})$ are relevant when toroidal and cylindrical effects are strongly dominant, respectively; the Hamaguchi-Horton parameter [16] follows from (9a)).

These conclusions are based on the analysis of reduced linear electrostatic two fluid equations which were derived under the assumption of standard drift wave ordering [14]. We discuss below the restrictions inherent to this approach:

(i) The wave-particle resonant interaction (ion Landau damping) has been qualitatively taken into account by assuming a low mode number cut-off of the unstable range at $(\omega-$ $\left.\omega_{E \times B}\right) / k_{\|} c_{i}=1$; other authors [16, 28] introduced an effective damping rate proportional to $k_{\|} c_{i}$ instead.

(ii) Trapped particle effects have not been considered; they could modify our conclusions in certain parameter ranges.

(iii) Electromagnetic effects may be important when the ratio of kinetic to magnetic pressure is sufficient or at low values of $\varepsilon_{N}$ [29]; worth noting is the fact that the electromagnetic corrections associated with small $k_{\|}$are expected to be reduced when toroidal effects play a leading role, since the sidebands have a larger parallel mode number than the primary oscillation: $\left(k_{\|}\right)_{ \pm 1} /\left(k_{\|}\right)_{0} \cong \Delta_{l, m} / w_{l, m} \gg 1$.

(iv) The standard drift wave ordering dissociates the equilibrium and oscillation length scales (their ratio $\rightarrow \infty$ ). However, as Eq. (5) shows, $\lim _{L_{N} \rightarrow 0} w_{l, m} \propto\left(L_{N}\right)^{-1 / 2}$ (assuming that $\eta_{i}-$ $2 / 3$ remains positive for instability), suggesting that the theory may break down when the profiles become too steep. 
These challenging extensions of the model will be investigated in a later work.

Although recent experiments with LHCD preheating show that a proper tailoring of the current profile during pre-heating and current ramp-up can significantly reduce the threshold power, we ought to note that ITBs have also been obtained experimentally and predicted by simulations in discharges without minimum $q[8,25]$. Moreover, ETBs have been observed in other circumstances [30, 31].

Note added in proof: We have only considered, in this article, the 'fundamental' ITG mode given by Eq. (4). The whole series of ITG radial eigenfunctions involves the Hermite polynomials $H_{n}$; the prerequisites set forth in the article being verified, the growth rate of the $n$th eigenmode increases with $n$ as $2 n+1$. For sufficiently large values of $n$, however, the term $(1+2 n) K_{t}$ will be larger than the term $\left(k_{\theta} a_{i}\right)^{2}$ on the left hand side of Eq. (I-70) of Ref. [14]. The premises assumed here are then no longer fulfilled, see the discussion preceding Eq. (4) and the remark following (5). In particular, the condition (I-94b) of negligible Landau damping on the sidebands that led to one of the inequalities (7) must be replaced by (I-87b). The latter shows that $\left|\operatorname{Re}\left(\omega-\omega_{\boldsymbol{E} \times \boldsymbol{B}}\right) / \mathrm{k}_{\|} \mathrm{c}_{\mathrm{i}}\right|_{\mathrm{p}= \pm 1} \rightarrow 0$ if the magnetic shear parameter tends to zero: ion Landau damping on the sidebands will stabilize the whole spectrum under those conditions.

\section{References}

[1] Levinton, F.M., et al., Phys. Rev. Lett. 75 (1995) 4417.

[2] Rice, B.W., et al., Phys. Plasmas 3 (1996) 1983.

[3] Fujita, T., et al., Phys. Rev. Lett. 78 (1997) 2377.

[4] Sips, A.C.C., et al., Plasma Phys. Control. Fusion 40 (1998) 1171.

[5] Bell, M.G., et al., Plasma Phys. Control. Fusion 41 (1999) A719.

[6] Günter, S., et al., Phys. Rev. Lett. 84 (2000) 3097.

[7] Zhu, P., Horton, W., Sugama, H., Phys. Plasmas 6 (1999) 2503.

[8] Zhu, P., Bateman, G., Kritz, A.H., Horton, W., Phys. Plasmas 7 (2000) 2898.

[9] Parail, V.V., JET Team, in Fusion Energy 2000 (Proc. 18th Int. Conf. Sorrento, 2000), CD-ROM, IAEA, Vienna (2001) CD-ROM file EXP5/05 and http://www.iaea.org/programmes/ripc/physics/ fec2000/html/node1.htm.
[10] Connor, J.W., Hastie, R.J., Taylor, J.B., Proc. R. Soc. London A. 365 (1979) 1.

[11] Romanelli, F., Zonca, F., Phys. Fluids B 5 (1993) 4081.

[12] Weiland, J., Collective Modes in Inhomogeneous Plasma, IOP Publishing, Bristol (2000).

[13] Hasselberg, G., Rogister, A., Plasma Phys. 22 (1980) 805.

[14] Rogister, A., Phys. Plasmas 7 (2000) 5070.

[15] Hahm, T.S., Burrell, K.H., Phys. Plasmas 2 (1995) 1648.

[16] Hamaguchi, S., Horton, W., Phys. Fluids B 4 (1992) 319.

[17] Hinton, F.L., Hazeltine, R.D., Rev. Mod. Phys. 48 (1976) 239.

[18] Balescu, R., Transport Processes in Plasmas, Vol. 2, Neoclassical Transport, North-Holland, Amsterdam (1988).

[19] Rogister, A., Phys. Rev. Lett. 81 (1998) 3663.

[20] Rogister, A., Phys. Plasmas 6 (1999) 200.

[21] Ongena, J., et al., Plasma Phys. Control. Fusion 41 (1999) A379.

[22] Rogister, A., Phys. Plasmas 2 (1995) 2729.

[23] Mazzucato, E., et al., Phys. Rev. Lett. 77 (1996) 3145 .

[24] Mazzucato, E., et al., in Fusion Energy 1996 (Proc. 16th Int. Conf. Montreal, 1996), Vol. 1, IAEA, Vienna (1997) 913.

[25] Guzdar, P.N., Liu, C.S., Dong, J.Q., Lee, Y.C., Phys. Rev. Lett. 57 (1986) 2818.

[26] Brunner, S., Fivaz, M., Tran, T.M., Vaclavik, J., Phys. Plasmas 5 (1998) 3929.

[27] Falchetto, G.L., Vaclavik, J., Maccio, M., Phys. Plasmas 7 (2000) 1196.

[28] Thyagaraja, A., Plasma Phys. Control. Fusion 42 (2000) B255.

[29] Zeiler, A., Drake, J.F., Rogers, B.N., Phys. Rev. Lett. 84 (2000) 99.

[30] Hogeweij, G.M.D., et al., Nucl. Fusion 38 (1998) 1881.

[31] Hoang, G.T., Phys. Rev. Lett. 84 (2000) 4593.

(Manuscript received 30 November 2000

Final manuscript accepted 30 March 2001)

E-mail address of A.L. Rogister:

a.rogister@fz-juelich.de

Subject classification: F2, Ti; D2, Te 